\title{
On the Growth of Meromorphic Solutions of Certain Nonlinear Difference Equations
}

\author{
Xiao-Min Li, Chen-Shuang Hao and Hong-Xun Yi
}

\begin{abstract}
By Cartan's version of Nevanlinna's theory, we prove the following result: let $m$ and $n$ be two positive integers satisfying $n \geq 2+m$, let $p \not \equiv 0$ be a polynomial, let $\eta \neq 0$ be a finite complex number, let $\omega_{1}, \omega_{2}, \ldots, \omega_{m}$ be $m$ distinct finite nonzero complex numbers, and let $H_{j}$ be either exponential polynomials of degree less than $q$, or an ordinary polynomial in $z$ for $0 \leq j \leq m$, such that $H_{j} \not \equiv 0$ for $1 \leq j \leq m$. Suppose that $f \not \equiv \infty$ is a meromorphic solution of the difference equation:

$$
\begin{aligned}
f^{n}(z)+p(z) f(z+\eta)= & H_{0}(z)+H_{1}(z) e^{\omega_{1} z^{q}}+H_{2}(z) e^{\omega_{2} z^{q}} \\
& +\cdots+H_{m}(z) e^{\omega_{m} z^{q}},
\end{aligned}
$$
\end{abstract}

such that the hyper-order of $f$ satisfies $\rho_{2}(f)<1$. Then, $f$ reduces to a transcendental entire function, such that either $n=m+2$ with $H_{0} \not \equiv 0$ and $\lambda(f)=\rho(f)=q$, or $m=2, H_{0}=0$ and:

$$
f(z)=\frac{H_{1}(z-\eta) e^{\omega_{1}(z-\eta)^{q}}}{p(z-\eta)}
$$

with

$$
H_{1}^{n}(z)=p^{n}(z) H_{2}(z+\eta) e^{\omega_{2} P_{q-1}(z)} \quad \text { and } \quad P_{q-1}(z)=\sum_{k=1}^{q}\left(\begin{array}{l}
q \\
k
\end{array}\right) \eta^{k} z^{q-k} .
$$

This result improves Theorems 1.1 and 1.3 from [19] by removing some assumptions of theirs. An example is provided to show that some results obtained in this paper, in a sense, are the best possible.

Mathematics Subject Classification. Primary 30D35; Secondary 39B32.

Keywords. Nevanlinna's theory, Cartan's version of Nevanlinna theory, difference Nevanlinna's theory, meromorphic functions, nonlinear difference equations.

\footnotetext{
Project was supported in part by the NSFC (no. 11171184), the NSF of Shandong Province, China (no. ZR2019MA029), and the FRFCU (no. 3016000841964007).
} 


\section{Introduction and Main Results}

In recent years, the difference variant of the Nevanlinna theory has been established in [5,8] and, in particular, in [7], by Halburd-Korhonen and by Chiang-Feng, independently. Using these theories, some mathematicians in the world began to consider the studies on the growth of meromorphic and entire solutions of certain nonlinear difference equations, and produced many fine works, for example, see $[13,16,18,19]$. In this paper, we will consider the growth of meromorphic solutions of the following nonlinear difference equation:

$f^{n}(z)+p(z) f(z+\eta)=H_{0}(z)+H_{1}(z) e^{\omega_{1} z^{q}}+H_{2}(z) e^{\omega_{2} z^{q}}+\cdots+H_{m}(z) e^{\omega_{m} z^{q}}$,

where $m$ and $n$ are the positive integers satisfying $n \geq m+2$, and $p \not \equiv 0$ is a polynomial, while $H_{j}$ is either an exponential polynomial of degree less than $q$ or an ordinary polynomial in $z$ for $0 \leq j \leq m$, while $\eta \neq 0$ is a finite complex value, and $\omega_{1}, \omega_{2}, \ldots, \omega_{m}$ are $m$ distinct finite nonzero complex numbers. Here, the notion of an exponential polynomial is defined as follows (cf. [11]): an exponential polynomial of order $q \geq 1$, which is an entire function of the form:

$$
g(z)=P_{1}(z) e^{Q_{1}(z)}+\cdots+P_{k}(z) e^{Q_{k}(z)},
$$

where $P_{j}$ and $Q_{j}$ are polynomials in $z$ for $1 \leq j \leq k$, such that $\max _{1 \leq j \leq k}$ $\left\{\operatorname{deg}\left(Q_{j}\right)\right\}=q$ (cf. [14]). Following Steinmetz [14], such a function can be written in the form:

$$
g(z)=H_{1}(z) e^{\omega_{1} z^{q}}+\cdots+H_{m}(z) e^{\omega_{m} z^{q}},
$$

where $\omega_{1}, \ldots, \omega_{m}(m \leq k)$ are pairwise different leading coefficients of the polynomials $Q_{1}, Q_{1}, \ldots$ and $Q_{k}$, and the function $H_{j}$ is either an exponential polynomial of order $\leq q-1$ or an ordinary polynomial in $z$ for $1 \leq j \leq k$. We use the convention that an exponential polynomial of order zero is an ordinary polynomial.

Throughout this paper, by meromorphic functions, we will always mean meromorphic functions in the complex plane. We described the standard notations of the Nevanlinna theory of meromorphic functions as in $[12,15,17]$. It will be convenient to let $E$ denote any set of positive real numbers of finite linear measure, not necessarily the same at each occurrence. For a nonconstant meromorphic function $h$, we denote by $T(r, h)$ the Nevanlinna characteristic of $h$ and by $S(r, h)$ any quantity satisfying $S(r, h)=o(T(r, h))$, as $r$ runs to infinity outside of a set of finite logarithmic measure. We say that $\alpha$ is a small function of $f$, if $\alpha$ is a meromorphic function, such that $T(r, \alpha)=S(r, f)$ (cf. [15]). A polynomial $P(z, f)$ is called a differential polynomial in $f$ whenever $f$ is a polynomial in $f$ and its derivatives, with small functions of $f$ as the coefficients. Similarly, a polynomial $Q(z, f)$ is called a differential-difference polynomial in $f$ whenever $Q(z, f)$ is a polynomial in $f$, its derivatives, and its shifts $f(z+\eta)$ with small functions of $f$ again as the coefficients (cf. [16]). In addition, we denote by $\rho(f), \rho_{2}(f), \lambda(f)$, and $\lambda_{2}(f)$ the order, the hyper-order, the exponent of convergence of zeros, and the hyper-exponent of convergence of zeros of $f$, respectively. Their definitions 
can be found in $[4,12]$. For convenience, we give their detailed definitions as follows:

Definition 1.1. For a nonconstant meromorphic function $f$, the order of $f$, the hyper-order of $f$, the exponent of convergence of zeros of $f$, and the hyperexponent of convergence of zeros of $f$ are denoted by $\rho(f), \rho_{2}(f), \lambda(f)$, and $\lambda_{2}(f)$, respectively. Their definitions are defined as:

$$
\begin{aligned}
\rho(f) & =\limsup _{r \rightarrow \infty} \frac{\log T(r, f)}{\log r}, \quad \rho_{2}(f)=\limsup _{r \rightarrow \infty} \frac{\log \log T(r, f)}{\log r}, \\
\lambda(f) & =\limsup _{r \rightarrow \infty} \frac{\log N\left(r, \frac{1}{f}\right)}{\log r}=\limsup _{r \rightarrow \infty} \frac{\log n\left(r, \frac{1}{f}\right)}{\log r} \text { and } \\
\lambda_{2}(f) & =\limsup _{r \rightarrow \infty} \frac{\log \log N\left(r, \frac{1}{f}\right)}{\log r}=\limsup _{r \rightarrow \infty} \frac{\log \log n\left(r, \frac{1}{f}\right)}{\log r} \text { respectively. }
\end{aligned}
$$

We recall the following results due to Yang-Laine [16]:

Theorem 1.2 ([16, Theorem 2.4]). Let $p$ and $q$ be polynomials. Then, a nonlinear difference equation:

$$
f^{2}(z)+q(z) f(z+1)=p(z)
$$

has no transcendental entire solution of finite order.

Theorem 1.3 ([16, Theorem 2.5]). A nonlinear difference equation:

$$
f^{3}(z)+q(z) f(z+1)=c \sin (b z),
$$

where $q$ is a nonconstant polynomial and $b, c \in \mathbb{C}$ are nonzero constants, do not admit entire solutions of finite order. If $q$ is a nonzero constant, then this equation possesses three distinct entire solutions of finite order, provided $b=3 n \pi$ and $q^{3}=(-1)^{n+1} \frac{27}{4} c^{2}$ for a nonzero integer $n$.

Recently, Zhang and Huang [19] proved the following result which extends Theorems 1.2 and 1.3:

Theorem 1.4 ([19, Theorem 1.1]). Let $s$ and $n$ be two positive integers satisfying $n \geq 2+s$, let $p \neq \equiv 0$ be a polynomial, let $\eta \neq 0$ be a finite complex number, let $\beta_{1}, \beta_{2}, \ldots, \beta_{s}$ be $s$ finite nonzero complex numbers, and let $\alpha_{1}, \alpha_{2}, \ldots, \alpha_{s}$ be $s$ distinct finite nonzero complex numbers. Suppose that $\frac{\alpha_{i}}{\alpha_{j}} \neq n$ for all $i, j \in\{1,2, \ldots, s\}$, and that $n \alpha_{k} \neq l_{k_{1}} \alpha_{1}+l_{k_{2}} \alpha_{2}+\cdots+l_{k_{s}} \alpha_{s}$ for $5 \leq k \leq s$ when $s \geq 5$, where $l_{k_{1}}, l_{k_{2}}, \ldots, l_{k_{s}} \in\{0,1,2, \ldots, n-1\}$ satisfying $l_{k_{1}}+l_{k_{2}}+\cdots+l_{k_{s}}=n$. Then, for any nonconstant meromorphic solution $f$ of the equation:

$$
f^{n}(z)+p(z) f(z+\eta)=\beta_{1}(z) e^{\alpha_{1} z}+\beta_{2}(z) e^{\alpha_{2} z}+\cdots+\beta_{s}(z) e^{\alpha_{s} z},
$$

we have $\rho_{2}(f) \geq 1$.

Theorem 1.5 ([19, Theorem 1.2]). Let $n$ and $s$ be two positive integers satisfying $n=s+1$, let $p \not \equiv 0$ be a polynomial, let $\eta \neq 0$ be a finite complex number, let $\beta_{1}, \beta_{2}, \ldots, \beta_{s}$ be finite nonzero complex numbers, and let $\alpha_{1}, \alpha_{2}, \ldots, \alpha_{s}$ be $s$ distinct finite nonzero complex numbers. Suppose that 
$\frac{\alpha_{i}}{\alpha_{j}} \neq n$ for all $i, j \in\{1,2, \ldots, s\}$. Then, any nonconstant meromorphic solution $f$ of Eq. (1.2) of hyper-order $\rho_{2}(f)<1$ must be an entire function, such that $\rho(f)=\lambda(f)=1$.

Regarding Theorems 1.4 and 1.5, one may ask the following two questions:

Question 1.6. What can be said about the conclusions of Theorem 1.4, if we remove the assumptions "Suppose that $\frac{\alpha_{i}}{\alpha_{j}} \neq n$ for all $i, j \in\{1,2, \ldots, s\}$, and that $n \alpha_{k} \neq l_{k_{1}} \alpha_{1}+l_{k_{2}} \alpha_{2}+\cdots+l_{k_{s}} \alpha_{s}$ for $5 \leq k \leq s$ when $s \geq 5$, where $l_{k_{1}}, l_{k_{2}}, \ldots, l_{k_{s}} \in\{0,1,2, \ldots, n-1\}$ satisfying $l_{k_{1}}+l_{k_{2}}+\cdots+l_{k_{s}}=n$ " in Theorem 1.4?

Question 1.7. What can be said about the conclusions of Theorem 1.5, if we remove the assumption "Suppose that $\frac{\alpha_{i}}{\alpha_{j}} \neq n$ for all $i, j \in\{1,2, \ldots, s\}$ " in Theorem 1.5?

In this direction, we first study the growth of a nonconstant meromorphic solution of Eq. (1.1) which is much more general difference equation than the difference equation (1.2). Indeed, we will prove the following result:

Theorem 1.8. Let $m$ and $n$ be two positive integers satisfying $n \geq 2+m$, let $p \not \equiv 0$ be a polynomial, let $\eta \neq 0$ be a finite complex number, let $\omega_{1}, \omega_{2}, \ldots, \omega_{m}$ be $m$ distinct finite nonzero complex numbers, let $H_{j}$ be either exponential polynomials of degree less than $q$, or an ordinary polynomials in $z$ for $0 \leq j \leq m$, and let $H_{j} \not \equiv 0$ for $1 \leq j \leq m$. Suppose that $f \not \equiv \infty$ is a meromorphic solution of the difference equation (1.1), such that the hyper-order of $f$ satisfies $\rho_{2}(f)<1$. Then, $f$ reduces to a transcendental entire function, such that one of the following two cases can occur:

(i) The positive integer $m$ in (1.1) satisfies $m=2$, and that the entire function $H_{0}$ in (1.1) satisfies $H_{0}=0$. Moreover, $f$ can be expressed as:

$$
f(z)=\frac{H_{1}(z-\eta) e^{\omega_{1}(z-\eta)^{q}}}{p(z-\eta)}
$$

with

$$
H_{1}^{n}(z)=p^{n}(z) H_{2}(z+\eta) e^{\omega_{2} P_{q-1}(z)} \quad \text { and } \quad P_{q-1}(z)=\sum_{k=1}^{q}\left(\begin{array}{l}
q \\
k
\end{array}\right) \eta^{k} z^{q-k} .
$$

(ii) The positive integers $m$ and $n$ in (1.1) satisfy $n=m+2$, and the entire function $H_{0}$ in (1.1) satisfies $H_{0} \not \equiv 0$. Moreover, the order of $f$ and the exponent of convergence of zeros of $f$ satisfy $\lambda(f)=\rho(f)=q$.

By Theorem 1.8, we can get the following two results, which solve Questions 1.6 and 1.7, respectively, and improve Theorems 1.4 and 1.5, respectively:

Corollary 1.9. Let $m$ and $n$ be two positive integers satisfying $n \geq 2+m$ and $m \neq 2$, let $p \not \equiv 0$ be a polynomial, let $\eta \neq 0$ be a finite complex number, let $\omega_{1}$, $\omega_{2}, \cdots, \omega_{m}$ be $m$ distinct finite nonzero complex numbers, let $H_{j}$ be either exponential polynomials of degree less than $q$, or an ordinary polynomials in 
$z$ for $1 \leq j \leq m$, and let $H_{j} \not \equiv 0$ for $1 \leq j \leq m$. Suppose that $f \not \equiv \infty$ is a meromorphic solution of the difference equation:

$$
f^{n}(z)+p(z) f(z+\eta)=H_{1}(z) e^{\omega_{1} z^{q}}+H_{2}(z) e^{\omega_{2} z^{q}}+\cdots+H_{m}(z) e^{\omega_{m} z^{q}} .
$$

Then, $f$ reduces to a transcendental entire function, such that its hyper-order $\rho_{2}(f)$ satisfies $\rho_{2}(f) \geq 1$.

By Theorem 1.8, we also get the following result, which is a supplement of Theorem 1.4 and Corollary 1.9:

Corollary 1.10. Let $m$ and $n$ be two positive integers satisfying $n \neq 2+m$, let $p \not \equiv 0$ be a polynomial, let $\eta \neq 0$ be a finite complex number, let $\omega_{1}, \omega_{2}, \ldots, \omega_{m}$ be $m$ distinct finite nonzero complex numbers, let $H_{j}$ be either exponential polynomials of degree less than $q$, or an ordinary polynomials in $z$ for $0 \leq$ $j \leq m$, and let $H_{j} \not \equiv 0$ for $0 \leq j \leq m$. Suppose that $f \not \equiv \infty$ is a meromorphic solution of the difference equation (1.1). Then, $f$ reduces to a transcendental entire function, such that the hyper-order of $f$ satisfies $\rho_{2}(f) \geq 1$.

The following example shows that the assumption of " $n \geq 2+m$ " in Corollary 1.9, in a sense, is the best possible. The example also shows that the assumption of " $H_{0} \not \equiv 0$ " in Corollary 1.10 is necessary.

Example 1.11. Let:

$$
f(z)=e^{\frac{z}{5}}+e^{-\frac{z}{5}}
$$

Then, it follows that the entire function $f$ defined as in (1.4) is an entire solution of the difference equation:

$$
f^{5}(z)-10 f(z+10 \pi i)=5 e^{\frac{3 z}{5}}+5 e^{-\frac{3 z}{5}}+e^{z}+e^{-z},
$$

such that $\rho(f)=1$ and:

$T(r, f(z))=N\left(r, \frac{1}{f(z)}\right)+O(1)=N\left(r, \frac{1}{e^{\frac{2 z}{5}}+1}\right)+O(1)=\frac{2 r}{5 \pi}(1+o(1))$.

Moreover, we can see that $n=m+1$ and $n=5$.

\section{Preliminaries}

In this section, we will introduce some results used to prove the main result in the present paper. First of all, we introduce the following result due to Yang and Yi [15].

Lemma 2.1 ([15, Theorem 1.62]). Let $f_{1}, f_{2}, \ldots, f_{n}$ be nonconstant meromorphic functions, and let $f_{n+1} \not \equiv 0$ be a meromorphic function such that $\sum_{j=1}^{n+1} f_{j}=1$. Suppose that there exists a subset $\mathrm{I} \subseteq \mathbb{R}^{+}$with linear measure mes I $=\infty$, such that:

$$
\sum_{i=1}^{n+1} N\left(r, \frac{1}{f_{i}}\right)+n \sum_{\substack{i=1 \\ i \neq j}}^{n+1} \bar{N}\left(r, f_{i}\right)<(\sigma+o(1)) T\left(r, f_{j}\right), \quad j=1,2, \ldots, n,
$$

as $r \in \mathrm{I}$ and $r \rightarrow \infty$, where $\sigma$ is a real number satisfying $0 \leq \sigma<1$. Then, $f_{n+1}=1$. 
For introducing the following lemma, we give the following notation (cf. [6, Definition 2.1]): for a meromorphic function $f$ satisfying $f \not \equiv 0$ and a positive integer $j$, we let $n_{j}(r, 1 / f)$ denote the number of zeros of $f$ in $\{z:|z| \leq r\}$, counted in the following manner: a zero of $f$ of multiplicity $m$ is counted exactly $k$ times where $k=\min \{m, j\}$. Then, we let $N_{j}(r, 1 / f)$ denote the corresponding integrated counting function, that is:

$$
N_{j}\left(r, \frac{1}{f}\right)=\int_{0}^{r} \frac{n_{j}\left(t, \frac{1}{f}\right)-n_{j}\left(0, \frac{1}{f}\right)}{t} \mathrm{~d} t+n_{j}\left(0, \frac{1}{f}\right) \log r .
$$

Obviously:

$$
N_{1}\left(r, \frac{1}{f}\right)=\bar{N}\left(r, \frac{1}{f}\right), \quad \bar{N}\left(r, \frac{1}{f}\right) \leq N_{j}\left(r, \frac{1}{f}\right) \leq N\left(r, \frac{1}{f}\right)
$$

and

$$
N_{j}\left(r, \frac{1}{f}\right) \leq j \bar{N}\left(r, \frac{1}{f}\right) .
$$

In 1933, Cartan proved a generalization of the second fundamental theorem, and for certain kinds of questions. Moreover, Cartan's theorem seems to give the better result than the second fundamental theorem. We now state the simple form of Cartan's theorem as follows.

Lemma 2.2 (Cantan's theorem, [3]). Let $g_{1}, g_{2}, \ldots, g_{p}$ be linearly independent entire functions, where $p \geq 2$. Suppose that for each complex number $z$, we have $\max \left\{\left|g_{1}(z)\right|,\left|g_{2}(z)\right|, \ldots,\left|g_{p}(z)\right|\right\}>0$. For positive $r$ :

$$
T(r)=\frac{1}{2 \pi} \int_{0}^{2 \pi} u\left(r e^{i \theta}\right) \mathrm{d} \theta-u(0), \quad \text { where } u(z)=\sup _{1 \leq j \leq p} \log \left|g_{j}(z)\right| \text {. }
$$

Set $g_{p+1}=g_{1}+g_{2}+\cdots+g_{p}$. Then, we have:

$$
T(r) \leq \sum_{j=1}^{p+1} N_{p-1}\left(r, \frac{1}{g_{j}}\right)+S(r) \leq(p-1) \sum_{j=1}^{p+1} \bar{N}\left(r, \frac{1}{g_{j}}\right)+S(r),
$$

where $S(r)$ is a quantity satisfying:

$$
S(r)=O(\log T(r))+O(\log r), \quad \text { as } r \rightarrow \infty \text { and } r \notin E .
$$

If at least one of the quotients $g_{j} / g_{m}$ is a transcendental function, then:

$$
S(r)=o(T(r)) \quad \text { as } r \rightarrow \infty \text { and } r \notin E,
$$

while if all the quotients $g_{j} / g_{m}$ are rational functions, then:

$$
S(r) \leq-\frac{1}{2} p(p-1) \log r+O(1), \quad \text { as } r \rightarrow \infty \text { and } r \notin E .
$$

Here, $E \subset(0,+\infty)$ is a subset of finite linear measure.

We also need the following result:

Lemma 2.3. [3] Assume that the hypotheses of Lemma 2.2 hold. Then, for any $j$ and $m$, we have:

$$
T\left(r, \frac{g_{j}}{g_{m}}\right) \leq T(r)+O(1), \quad \text { as } r \rightarrow \infty,
$$


and for any $j$, we have:

$$
N\left(r, \frac{1}{g_{j}}\right) \leq T(r)+O(1), \quad \text { as } r \rightarrow \infty .
$$

For introducing the following two lemmas, we introduce the notion of the convex hull of a subset of the complex plane $\mathbb{C}$ (cf. [14]): the convex hull of a subset $W \subset \mathbb{C}$, denoted as $\operatorname{co}(\mathrm{W})$, is the intersection of all convex sets containing the set $W$. If $W$ contains only finitely many elements, then $\operatorname{co}(\mathrm{W})$ is obtained as an intersection of finitely many closed half-planes, and hence, $\mathrm{co}(\mathrm{W})$ is either a compact polygon (with a nonempty interior) or a line segment. We denote the perimeter of $\operatorname{co}(\mathrm{W})$ by $\mathrm{C}(\operatorname{co}(\mathrm{W}))$. If $\operatorname{co}(\mathrm{W})$ is a line segment, then $\mathrm{C}(\mathrm{co}(\mathrm{W}))$ equals to twice the length of this line segment. Throughout the rest of the paper, we fix the notations for $W=\left\{\bar{\omega}_{1}, \bar{\omega}_{2}, \ldots, \bar{\omega}_{m}\right\}$ and $W_{0}=\left\{0, \bar{\omega}_{1}, \bar{\omega}_{2}, \ldots, \bar{\omega}_{m}\right\}$.

Lemma 2.4 ([14, Satz 1 and Satz 2$])$. Let $f$ be given as:

$$
f(z)=H_{0}(z)+H_{1}(z) e^{\omega_{1} z^{q}}+H_{2}(z) e^{\omega_{2} z^{q}}+\cdots+H_{m}(z) e^{\omega_{m} z^{q}},
$$

where $m$ and $q$ are positive integers, $H_{j}$ is either an exponential polynomial of degree less than $q$ or an ordinary polynomial in $z$ for $0 \leq j \leq m$, and that $\omega_{1}, \omega_{2}, \ldots, \omega_{m}$ are $m$ distinct finite nonzero complex numbers. Suppose that $H_{j} \not \equiv 0$ for $1 \leq j \leq m$. Then:

$$
T(r, f)=C\left(\operatorname{co}\left(W_{0}\right)\right) \frac{r^{q}}{2 \pi}+o\left(r^{q}\right) .
$$

If $H_{0} \not \equiv 0$, then:

$$
m\left(r, \frac{1}{f}\right)=o\left(r^{q}\right) .
$$

If $H_{0}=0$, then:

$$
N\left(r, \frac{1}{f}\right)=C\left(\operatorname{co}\left(W_{0}\right)\right) \frac{r^{q}}{2 \pi}+o\left(r^{q}\right) .
$$

The following result is due to Halburd et al. [9]:

Lemma $2.5([9$, Lemma 8.3]). Let $T(r):[0,+\infty) \rightarrow[0,+\infty)$ be a nondecreasing continuous function and let $s \in \mathbb{R}^{+}$. If the hyper-order of $T$ is strictly less than one, i.e., $\lim \sup _{r \rightarrow \infty} \frac{\log \log T(r)}{\log r}=\zeta<1$, and let $\delta \in(0,1-\zeta)$, then $T(r+s)=T(r)+o\left(\frac{T(r)}{r^{\delta}}\right)$, where $r$ runs to infinity outside of a set of finite logarithmic measure.

The following result is proved by Chiang and Feng [5]:

Lemma 2.6 ([5, Theorem 2.1]). Let $f$ be a meromorphic function of order $\rho(f)=\rho<\infty$, and let $\eta$ be a fixed nonzero complex number, and then, for each $\varepsilon>0$, we have:

$$
T(r, f(z+\eta))=T(r, f(z))+O\left(r^{\rho-1+\varepsilon}\right)+O(\log r) .
$$




\section{Proof of Theorem 1.8}

First, for convenience, we give the following notation of some assumptions of Theorem 1.8:

(H). Suppose that $H_{0}$ and $H_{j} \not \equiv 0$ are either exponential polynomials of degree less than $q$, or the ordinary polynomials in $z$ for $1 \leq j \leq m$, and that $\omega_{1}, \omega_{2}, \ldots, \omega_{m}$ are $m$ distinct finite nonzero complex numbers.

By noting that $f \not \equiv \infty$ is a meromorphic solution of (1.1), we consider the following two cases.

Case 1. Suppose that $f$ is a constant, say $f=c$, where $c$ is some finite constant. Then, by rewriting (1.1), we have:

$$
H_{1}(z) e^{\omega_{1} z^{q}}+H_{2}(z) e^{\omega_{2} z^{q}}+\cdots+H_{m}(z) e^{\omega_{m} z^{q}}=c^{n}+c p(z)-H_{0}(z) .
$$

Clearly, the left hand of (3.1) is an exponential polynomial of order $q$ and the right hand of (3.1) is an exponential polynomial of order less than $q$, or an ordinary polynomial in $z$, this is a contradiction. Thus, $c^{n}+c p-H_{0}=0$. This together with (3.1) gives:

$$
H_{1}(z) e^{\omega_{1} z^{q}}+H_{2}(z) e^{\omega_{2} z^{q}}+\cdots+H_{m}(z) e^{\omega_{m} z^{q}}=0 .
$$

By dividing $e^{\omega_{1} z^{q}}$ on two sides of (3.2), we have:

$$
H_{2}(z) e^{\left(\omega_{2}-\omega_{1}\right) z^{q}}+\cdots+H_{m}(z) e^{\left(\omega_{m}-\omega_{1}\right) z^{q}}=-H_{1}(z) .
$$

In the same manner as the above, we deduce $-H_{1}=0$, which contradicts the assumption $H_{1} \not \equiv 0$.

Case 2. Suppose that $f$ is a nonconstant meromorphic function such that $\rho_{2}(f)<1$. First of all, we prove that $f$ is a transcendental entire function. Indeed, we suppose that $z_{0} \in \mathbb{C}$ is a pole of $f(z)$ of multiplicity equal to $\nu$. Then, it follows by (1.1) and the assumption of Theorem 1.8 that $z_{0}+\eta$ is a pole of $f(z)$ of multiplicity equal to $n \nu$ at least. By substituting $z=z_{0}+\eta$ into (1.1), we have:

$$
f^{n}\left(z_{0}+\eta\right)+p\left(z_{0}+\eta\right) f(z+2 \eta)=H_{0}\left(z_{0}+\eta\right)+\sum_{j=1}^{m} H_{j}\left(z_{0}+\eta\right) e^{\omega_{j}\left(z_{0}+\eta\right)^{q}},
$$

which implies that $z_{0}+2 \eta$ is a pole of $f(z)$ of multiplicity equal to $n^{2} \nu$ at least. Following this step, we can find a sequence $\left\{z_{0}+j \eta\right\}_{j=0}^{\infty}$ of the poles of $f(z)$. Here, $z_{0}+j \eta$ is a pole of $f(z)$ of multiplicity equal to $n^{j} \nu$ for each positive integer $j$. Therefore, for each positive integer $j$, we have:

$$
n\left(j|\eta|+\left|z_{0}\right|+1, f\right) \geq \nu+n \nu+\cdots+n^{j} \nu,
$$

which together with the assumption $n \geq m+2 \geq 3$ gives:

$$
\begin{aligned}
\lambda_{2}\left(\frac{1}{f}\right) & =\limsup _{r \rightarrow \infty} \frac{\log \log n(r, f)}{\log r} \geq \limsup _{j \rightarrow \infty} \frac{\log \log n\left(j|\eta|+\left|z_{0}\right|+1, f(z)\right)}{\log \left(j|\eta|+\left|z_{0}\right|+1\right)} \\
& \geq \limsup _{j \rightarrow \infty} \frac{\log \log n^{j}}{\log \left(j|\eta|+\left|z_{0}\right|+1\right)}=\limsup _{j \rightarrow \infty} \frac{\log j+\log \log n}{\log \left(j|\eta|+\left|z_{0}\right|+1\right)}=1 .
\end{aligned}
$$


On the other hand, by Definition 1.1, we have $\rho_{2}(f) \geq \lambda_{2}\left(\frac{1}{f}\right)$. This together with (3.4) gives $\rho_{2}(f) \geq 1$, which contradicts the assumption $\rho_{2}(f)<1$. Therefore, $f$ is a nonconstant entire function of hyper-order $\rho_{2}(f)<1$. We consider the following two subcases:

Subcase 2.1 Suppose that $f$ is a nonconstant polynomial. Then:

$$
T(r, f)=O(\log r) .
$$

By rewriting (1.1), we have:

$H_{1}(z) e^{\omega_{1} z^{q}}+H_{2}(z) e^{\omega_{2} z^{q}}+\cdots+H_{m}(z) e^{\omega_{m} z^{q}}=f^{n}(z)+p(z) f(z+\eta)-H_{0}(z)$.

By (3.5) and the assumption $(\mathrm{H})$, we deduce for $1 \leq j \leq m$ and $1 \leq k \leq m$ that:

$$
T\left(r, H_{j}(z)\right)+T\left(r, f^{n}(z)+p(z) f(z+\eta)-H_{0}(z)\right)=o\left(T\left(r, e^{\omega_{k} z^{q}}\right),\right.
$$

as $r \rightarrow \infty$.

By (3.6), (3.7) and Lemma 2.1, we deduce (3.2) and:

$$
f^{n}(z)+p(z) f(z+\eta)-H_{0}(z)=0 .
$$

By (3.8), we see that (3.6) can be rewritten as (3.2). By dividing $e^{\omega_{1} z^{q}}$ on two sides of (3.2), we have (3.3). By (3.3), (3.7), Lemma 2.1, the assumption $(\mathrm{H})$ and Hayman $[10, \mathrm{p} .7]$, we deduce $H_{1}=0$, which is impossible.

Subcase 2.2 Suppose that $f$ is a transcendental entire function. We consider the following two subcases.

Subcase 2.2.1. Suppose that $H_{0}=0$. Then, (1.1) can be rewritten as:

$$
f^{n}(z)=H_{1}(z) e^{\omega_{1} z^{q}}+H_{2}(z) e^{\omega_{2} z^{q}}+\cdots+H_{m}(z) e^{\omega_{m} z^{q}}-p(z) f(z+\eta) \text {. }
$$

By the assumption $(\mathrm{H})$, we deduce that $H_{1}(z) e^{\omega_{1} z^{q}}, H_{2}(z) e^{\omega_{2} z^{q}}, \ldots$, $H_{m}(z) e^{\omega_{m} z^{q}}$ are $m$ distinct linearly independent entire functions. Combining this with (3.9), we consider the following two subcases:

Subcase 2.2.1.1. Suppose that $H_{1}(z) e^{\omega_{1} z^{q}}, H_{2}(z) e^{\omega_{2} z^{q}}, \ldots, H_{m}(z) e^{\omega_{m} z^{q}}$, $-p(z) f(z+\eta)$ are $m+1$ linearly dependent entire functions in the complex plane. Then, $p(z) f(z+\eta)$ can be linearly expressed by the $m$ distinct entire functions $H_{1}(z) e^{\omega_{1} z^{q}}, H_{2}(z) e^{\omega_{2} z^{q}}, \ldots, H_{m}(z) e^{\omega_{m} z^{q}}$. This means that there exist $m$ finite complex constants $k_{1}, k_{2}, \ldots, k_{m}$, such that:

$$
p(z) f(z+\eta)=\sum_{j=1}^{m} k_{j} H_{j}(z) e^{\omega_{j} z^{q}} .
$$

By substituting (3.10) into (3.9), we deduce:

$$
f^{n}(z)=\sum_{j=1}^{m}\left(1-k_{j}\right) H_{j}(z) e^{\omega_{j} z^{q}} .
$$

Without loss of generality, we suppose that $1-k_{j} \neq 0$ for $1 \leq j \leq m$. Moreover, by the assumption $(\mathrm{H})$, we deduce that the $m$ distinct entire functions:

$$
\left(1-k_{1}\right) H_{1}(z) e^{\omega_{1} z^{q}},\left(1-k_{2}\right) H_{2}(z) e^{\omega_{2} z^{q}}, \ldots,\left(1-k_{m}\right) H_{m}(z) e^{\omega_{m} z^{q}}
$$

in (3.11) are linearly independent transcendental entire functions. 
Suppose that $f^{n}(z)$ and the $m$ distinct entire functions in (3.12) have infinitely many common zeros $a_{1,1}, a_{1,2}, \ldots, a_{1, k}, \ldots$, in the complex plane, such that $a_{1, k} \rightarrow \infty$. Then, by Weierstrass's factorization theorem (cf. [2, Theorem 7 in Chapter 5, p.195]), we can find that there exists an entire function $\gamma_{1}(z)$ with the common zeros of $f^{n}(z)$ and the $m$ distinct entire functions in (3.12) for zeros, where each such common zero $a_{1, k} \in\left\{a_{1, k}\right\}_{k=1}^{\infty}$ is counted according to the minimum of all the multiplicities of $a_{1, k}$ as the zero of $f^{n}(z)$ and the $m$ distinct entire functions in (3.12), respectively, such that:

$$
\gamma_{1}(z)=z^{m_{1,0}} e^{g(z)} \prod_{k=1}^{\infty}\left(1-\frac{z}{a_{1, k}}\right) e^{\frac{z}{a_{1, k}}+\frac{1}{2}\left(\frac{z}{a_{1, k}}\right)^{2}+\cdots+\frac{1}{m_{1, k}}\left(\frac{z}{a_{1, k}}\right)^{m_{1, k}}},
$$

where the product is taken over all $a_{1, k} \neq 0$. Here, $m_{1,0}, m_{1,1}, \ldots, m_{1, k}, \ldots$, are certain nonnegative integers, and $g(z)$ is a constant or a nonconstant polynomial of degree less than or equal to $q-1$. Combining this with the assumption $(\mathrm{H})$, we deduce:

$$
N\left(r, \frac{1}{\gamma_{1}}\right) \leq \sum_{j=1}^{m} N\left(r, \frac{1}{H_{j}}\right) \leq \sum_{j=1}^{m} T\left(r, H_{j}\right)+O(1) \leq O\left(r^{q-1}\right) .
$$

By (3.14) and Ash [1, Theorem 4.3.6], we have:

$$
\lambda\left(\gamma_{1}\right)=\rho\left(\gamma_{1}\right) \leq q-1
$$

By dividing $\gamma_{1}$ defined as in (3.13) on two sides of (3.11), we have:

$$
\frac{f^{n}(z)}{\gamma_{1}(z)}=\sum_{j=1}^{m}\left(1-k_{j}\right) H_{1, j}(z) e^{\omega_{j} z^{q}}
$$

where:

$$
H_{1, j}(z)=\frac{H_{j}(z)}{\gamma_{1}(z)}, \quad 1 \leq j \leq m .
$$

Then, by (3.17) and the obtained result that the $m$ distinct transcendental entire functions in (3.12) are linearly independent in the complex plane, we deduce that the $m$ entire functions $\left(1-k_{1}\right) H_{1,1}(z) e^{\omega_{1} z^{q}},\left(1-k_{2}\right) H_{1,2}(z) e^{\omega_{2} z^{q}}$, $\ldots,\left(1-k_{m}\right) H_{1, m}(z) e^{\omega_{m} z^{q}}$ on the right-hand side of (3.16) are still linearly independent in the complex plane. Moreover, by (3.16) and the definition of the entire function $\gamma_{1}$ in $(3.13)$, we can deduce that the entire function $\frac{f^{n}(z)}{\gamma_{1}(z)}$ and the $m$ entire functions $\left(1-k_{1}\right) H_{1,1}(z) e^{\omega_{1} z^{q}},\left(1-k_{2}\right) H_{1,2}(z) e^{\omega_{2} z^{q}}, \ldots,(1-$ $\left.k_{m}\right) H_{1, m}(z) e^{\omega_{m} z^{q}}$ on the right-hand side of (3.16) have no common zeros in the complex plane. Furthermore, by (3.15), (3.17), the assumption $(\mathrm{H})$, and Hayman [10, p.7], we deduce:

$$
\frac{H_{1, i}(z)}{H_{1, j}(z)} e^{\left(\omega_{i}-\omega_{j}\right) z^{q}}=\frac{H_{i}(z)}{H_{j}(z)} e^{\left(\omega_{i}-\omega_{j}\right) z^{q}}
$$

are transcendental meromorphic functions for $1 \leq i<j<m$. For convenience, now, we set:

$$
\frac{f^{n}(z)}{\gamma_{1}(z)}=g_{1, m+1}(z)
$$


and

$$
\left(1-k_{j}\right) H_{1, j}(z) e^{\omega_{j} z^{q}}=g_{1, j}(z) \quad \text { for } \quad 1 \leq j \leq m .
$$

Then, it follows by (3.19), (3.20) and the above analysis that (3.16) can be rewritten as:

$$
g_{1, m+1}=\sum_{j=1}^{m} g_{1, j}
$$

such that $\max _{1 \leq j \leq m}\left\{\left|g_{1, j}(z)\right|\right\}>0$ for each $z \in \mathbb{C}$, and $g_{1,1}, g_{1,2}, \ldots, g_{1, m}$ in (3.21) are $m$ linearly independent transcendental entire functions. Therefore, by (3.15), (3.17), (3.18)-(3.21) and Lemmas 2.2 and 2.3, we deduce:

$$
\begin{aligned}
n N\left(r, \frac{1}{f}\right) & =N\left(r, \frac{1}{g_{1, m+1}}\right)+O\left(r^{q-1}\right) \leq T_{1}(r)+O\left(r^{q-1}\right) \\
& \leq \sum_{k=1}^{m+1} N_{m-1}\left(r, \frac{1}{g_{1, k}}\right)+O\left(r^{q-1}\right)+S_{1}(r) \\
& \leq(m-1) \bar{N}\left(r, \frac{1}{f}\right)+O\left(r^{q-1}\right)+S_{1}(r) \\
& \leq(m-1) N\left(r, \frac{1}{f}\right)+O\left(r^{q-1}\right)+S_{1}(r),
\end{aligned}
$$

where

$$
T_{1}(r)=\frac{1}{2 \pi} \int_{0}^{2 \pi} u_{1}\left(r e^{i \theta}\right) \mathrm{d} \theta-u_{1}(0) \quad \text { with } \quad u_{1}(z)=\sup _{1 \leq k \leq m}\left\{\log \left|g_{1, k}(z)\right|\right\},
$$

and $S_{1}(r)$ is a quantity satisfying::

$$
S_{1}(r)=O\left(\log T_{1}(r)\right)+O(\log r),
$$

as $r \rightarrow \infty$ and $r \notin E$. Here, $E \subset(0,+\infty)$ is a subset of finite linear measure.

By (3.22) and the assumption $n \geq m+2$, we deduce:

$$
N\left(r, \frac{1}{f}\right)=O\left(r^{q-1}\right)+S_{1}(r)
$$

and

$$
T_{1}(r) \leq(m-1) N\left(r, \frac{1}{f}\right)+O\left(r^{q-1}\right)+S_{1}(r) \leq(m-1) T(r, f)+O\left(r^{q-1}\right)+S_{1}(r),
$$

as $r \notin E$ and $r \rightarrow+\infty$. Since $f$ is a transcendental entire function, by (3.23) and (3.25), we deduce:

$$
S_{1}(r)=o(T(r, f))+O\left(r^{q-1}\right),
$$

as $r \notin E$ and $r \rightarrow+\infty$.

By (3.24) and (3.26), we deduce:

$$
N\left(r, \frac{\gamma_{1}(z) e^{\omega_{1} z^{q}}}{f^{n}(z)}\right) \leq n N\left(r, \frac{1}{f(z)}\right) \leq O\left(r^{q-1}\right)+o(T(r, f(z)))=o\left(r^{q}\right),
$$

as $r \notin E$ and $r \rightarrow+\infty$. 
On the other hand, by dividing $e^{\omega_{1} z^{q}}$ on two sides of (3.16), we have:

$$
\frac{f^{n}(z)}{\gamma_{1}(z) e^{\omega_{1} z^{q}}}=\left(1-k_{1}\right) H_{1,1}(z)+\sum_{j=2}^{m}\left(1-k_{j}\right) H_{1, j}(z) e^{\left(\omega_{j}-\omega_{1}\right) z^{q}} .
$$

By the assumption $(\mathrm{H})$ and the supposition $\left(1-k_{j}\right) H_{1, j} \not \equiv 0$ for $1 \leq j \leq m$, we deduce by (3.15), (3.17), (3.24)-(3.28), Lemma 2.4, and the first fundamental theorem that:

$$
\begin{aligned}
m\left(r, \frac{\gamma_{1}(z) e^{\omega_{1} z^{q}}}{f^{n}(z)}\right) & =m\left(r, \frac{1}{\left(1-k_{1}\right) H_{1,1}(z)+\sum_{j=2}^{m}\left(1-k_{j}\right) H_{1, j}(z) e^{\left(\omega_{j}-\omega_{1}\right) z^{q}}}\right) \\
& =o\left(r^{q}\right)
\end{aligned}
$$

and

$$
\begin{aligned}
N\left(r, \frac{\gamma_{1}(z) e^{\omega_{1} z^{q}}}{f^{n}(z)}\right)= & N\left(r, \frac{1}{\left(1-k_{1}\right) H_{1,1}(z)+\sum_{j=2}^{m}\left(1-k_{j}\right) H_{1, j}(z) e^{\left(\omega_{j}-\omega_{1}\right) z^{q}}}\right) \\
= & T\left(r, \frac{1}{\left(1-k_{1}\right) H_{1,1}(z)+\sum_{j=2}^{m}\left(1-k_{j}\right) H_{1, j}(z) e^{\left(\omega_{j}-\omega_{1}\right) z^{q}}}\right) \\
& +o\left(r^{q}\right) \\
= & T\left(r, \frac{\gamma_{1}(z) e^{\omega_{1} z^{q}}}{f^{n}(z)}\right)+o\left(r^{q}\right)=C\left(\operatorname{co}\left(\hat{W}_{0}\right)\right) \frac{r^{q}}{2 \pi}+o\left(r^{q}\right), \quad(3.29)
\end{aligned}
$$

where $\hat{W}_{0}=\left\{0, \overline{\omega_{2}-\omega_{1}}, \overline{\omega_{3}-\omega_{1}}, \ldots, \overline{\omega_{m}-\omega_{1}}\right\}$.

By (3.29) and (3.27), we get a contradiction.

Suppose that $f^{n}(z)$ and the $m$ distinct entire functions in (3.12) have at most finitely many common zeros in the complex plane, say that $a_{1,1}, a_{1,2}, \ldots$, $a_{1, l_{1}}$ are all the distinct common nonzero zeros of $f^{n}(z)$ and the $m$ distinct transcendental entire functions of (3.12) in the complex plane, where $l_{1}$ is a positive integer, and that each such common zero $a_{1, j} \in\left\{a_{1, j}\right\}_{j=1}^{l_{1}}$ is counted according to the minimum positive integer $\tilde{m}_{1, j}$ of all the multiplicities of $a_{1, j}$ as the zero of $f^{n}(z)$ and the $m$ distinct transcendental entire functions of (3.12), respectively. Now, we let:

$$
P_{1,1}(z)=z^{m_{1,0}} \prod_{j=1}^{l_{1}}\left(z-a_{1, j}\right)^{\tilde{m}_{1, j}},
$$

where $m_{1,0}$ is the nonnegative integer that is defined as in (3.13). Next, we replace the polynomial $P_{1,1}$ defined as in (3.30) with the entire function $\gamma_{1}$ defined as in (3.13), and then use the lines as the above, we can get a contradiction.

Subcase 2.2.1.2. Suppose that:

$$
H_{1}(z) e^{\omega_{1} z^{q}}, \quad H_{2}(z) e^{\omega_{2} z^{q}}, \quad \ldots, H_{m}(z) e^{\omega_{m} z^{q}},-p(z) f(z+\eta)
$$

are $m+1$ linearly independent entire functions in the complex plane.

Suppose that $f^{n}(z)$ and the $m+1$ distinct entire functions in (3.31) have infinitely many common zeros $a_{2,1}, a_{2,2}, \ldots, a_{2, k}, \ldots$, in the complex plane such that $a_{2, k} \rightarrow \infty$. Then, by Weierstrass's factorization theorem (cf. [2, Theorem 7 in Chapter 5, p.195]), we can find that there exists an entire 
function $\gamma_{2}(z)$ with the common zeros of $f^{n}(z)$ and the $m+1$ distinct entire functions in (3.31) for zeros, where each such common zero $a_{2, k} \in\left\{a_{2, k}\right\}_{k=1}^{\infty}$ is counted according to the minimum of all the multiplicities of $a_{2, k}$ as the zero of $f^{n}(z)$ and the $m+1$ distinct entire functions in (3.31), respectively, such that:

$$
\gamma_{2}(z)=z^{m_{2,0}} e^{\hat{g}(z)} \prod_{k=1}^{\infty}\left(1-\frac{z}{a_{2, k}}\right) e^{\frac{z}{a_{2, k}}+\frac{1}{2}\left(\frac{z}{a_{2, k}}\right)^{2}+\cdots+\frac{1}{m_{2, k}}\left(\frac{z}{a_{2, k}}\right)^{m_{2, k}}},
$$

where the product is taken over all $a_{2, k} \neq 0$. Here, $m_{2,0}, m_{2,1}, \ldots, m_{2, n}, \ldots$, are certain nonnegative integers, and $\hat{g}(z)$ is a finite constant or a nonconstant polynomial of degree less than or equal to $q-1$. Combining this with the assumption $(\mathrm{H})$, we deduce:

$$
N\left(r, \frac{1}{\gamma_{2}}\right) \leq \sum_{j=1}^{m} N\left(r, \frac{1}{H_{j}}\right) \leq \sum_{j=1}^{m} T\left(r, H_{j}\right)+O(1) \leq O\left(r^{q-1}\right)+O(\log r) .
$$

By (3.32), (3.33) and Ash [1, Theorem 4.3.6], we have:

$$
\lambda\left(\gamma_{2}\right)=\rho\left(\gamma_{2}\right) \leq q-1
$$

By dividing $\gamma_{2}$ defined as in (3.32) on two sides of (3.9), we have:

$$
\frac{f^{n}(z)}{\gamma_{2}(z)}=\sum_{j=1}^{m} H_{2, j}(z) e^{\omega_{j} z^{q}}-\frac{p(z) f(z+\eta)}{\gamma_{2}(z)},
$$

where:

$$
H_{2, j}(z)=\frac{H_{j}(z)}{\gamma_{2}(z)} \quad \text { for } \quad 1 \leq j \leq m .
$$

Then, by (3.35) and the supposition that the $m+1$ distinct entire functions in (3.31) are linearly independent in the complex plane, we deduce that $-\frac{p(z) f(z+\eta)}{\gamma_{2}(z)}$ and the $m$ distinct entire functions $H_{2,1}(z) e^{\omega_{1} z^{q}}, H_{2,2}(z) e^{\omega_{2} z^{q}}, \ldots$, $H_{2, m}(z) e^{\omega_{m} z^{q}}$ on the right-hand side of (3.35) are still linearly independent in the complex plane. Moreover, by (3.35) and the definition of the entire function $\gamma_{2}$ as in (3.32), we can deduce that the entire function $\frac{f^{n}(z)}{\gamma_{2}(z)}$ and the $m+1$ entire functions $H_{2,1}(z) e^{\omega_{1} z^{q}}, H_{2,2}(z) e^{\omega_{2} z^{q}}, \ldots, H_{2, m}(z) e^{\omega_{m} z^{q}},-\frac{p(z) f(z+\eta)}{\gamma_{2}(z)}$ on the right-hand side of (3.35) have no common zeros in the complex plane. Furthermore, by (3.36) and the assumption $(\mathrm{H})$, we deduce:

$$
\frac{H_{2, i}(z)}{H_{2, j}(z)} e^{\left(\omega_{i}-\omega_{j}\right) z^{q}}=\frac{H_{i}(z)}{H_{j}(z)} e^{\left(\omega_{i}-\omega_{j}\right) z^{q}}
$$

is a transcendental meromorphic function for $1 \leq i<j \leq m$. For convenience, now, we set:

$$
\begin{aligned}
\frac{f^{n}(z)}{\gamma_{2}(z)} & =g_{2, m+2}(z), \\
-\frac{p(z) f(z+\eta)}{\gamma_{2}(z)} & =g_{2, m+1}(z)
\end{aligned}
$$


and

$$
H_{2, j}(z) e^{\omega_{j} z^{q}}=g_{2, j}(z) \text { for } \quad 1 \leq j \leq m .
$$

Then, it follows by (3.38)-(3.40) that (3.35) can be rewritten as:

$$
g_{2, m+2}=\sum_{j=1}^{m+1} g_{2, j}
$$

such that $\max _{1 \leq j \leq m+1}\left\{\left|g_{2, j}(z)\right|\right\}>0$ for each $z \in \mathbb{C}$, and $g_{2,1}, g_{2,2}, \ldots, g_{2, m}$, $g_{2, m+1}$ on the right-hand side of (3.41) are $m+1$ linearly independent transcendental entire functions. By geometry observation, we deduce from Lemma 2.5 and the supposition $\rho_{2}(f)<1$ that:

$$
N\left(r, \frac{1}{f(z+\eta)}\right) \leq N\left(r+|\eta|, \frac{1}{f(z)}\right) \leq N\left(r, \frac{1}{f(z)}\right)+o\left(\frac{T(r, f(z))}{r^{\delta}}\right)
$$

and

$$
T(r, f(z+\eta)) \leq T(r+|\eta|, f(z)) \leq T(r, f(z))+o\left(\frac{T(r, f(z))}{r^{\delta}}\right),
$$

as $r \notin E_{1}$ and $r \rightarrow \infty$. Here, $E_{1} \subset(0,+\infty)$ is a subset of finite logarithmic measure, while $\delta$ is a fixed positive number satisfying $\delta \in\left(0,1-\rho_{2}\right)$ with $\rho_{2}$ being a real number, such that $\rho_{2}(f)=\limsup _{r \rightarrow \infty} \frac{\log \log T(r, f)}{\log r}=\rho_{2}<1$.

By (3.33), (3.37)-(3.42), Lemmas 2.2, 2.3, and Hayman [10, p.7], we deduce:

$$
\begin{aligned}
n N\left(r, \frac{1}{f(z)}\right) & =N\left(r, \frac{1}{g_{2, m+1}(z)}\right)+O\left(r^{q-1}\right) \leq T_{2}(r)+O\left(r^{q-1}\right) \\
& \leq \sum_{k=1}^{m+2} N_{m}\left(r, \frac{1}{g_{2, k}(z)}\right)+O\left(r^{q-1}\right)+S_{2}(r) \\
& \leq m \bar{N}\left(r, \frac{1}{f(z)}\right)+N\left(r, \frac{1}{f(z+\eta)}\right)+O\left(r^{q-1}\right)+O(\log r)+S_{2}(r) \\
& \leq m N\left(r, \frac{1}{f(z)}\right)+N\left(r, \frac{1}{f(z+\eta)}\right)+O\left(r^{q-1}\right)+O(\log r)+S_{2}(r) \\
& \leq m N\left(r, \frac{1}{f(z)}\right)+N\left(r+|\eta|, \frac{1}{f(z)}\right)+O\left(r^{q-1}\right)+O(\log r)+S_{2}(r) \\
& =(m+1) N\left(r, \frac{1}{f(z)}\right)+o\left(\frac{T(r, f(z))}{r^{\delta}}\right)+O\left(r^{q-1}\right)+O(\log r)+S_{2}(r)
\end{aligned}
$$

as $r \notin E_{1} \cup E$ and $r \rightarrow \infty$. Here:

$$
T_{2}(r)=\frac{1}{2 \pi} \int_{0}^{2 \pi} u_{2}\left(r e^{i \theta}\right) \mathrm{d} \theta-u_{2}(0) \quad \text { with } u_{2}(z)=\sup _{1 \leq k \leq m+1}\left\{\log \left|g_{2, k}(z)\right|\right\}
$$

and $S_{2}(r)$ is a quantity satisfying:

$$
S_{2}(r)=O\left(\log T_{2}(r)\right)+O(\log r)
$$

as $r \notin E_{1} \cup E$ and $r \rightarrow \infty$, where $E \subset(0,+\infty)$ is a subset of finite linear measure. 
By (3.44)-(3.46) and the assumption $n \geq m+2$, we deduce:

$$
N\left(r, \frac{1}{f(z)}\right)=O\left(r^{q-1}\right)+O\left(\log T_{2}(r)\right)+O(\log r)+S_{2}(r)
$$

and

$$
T_{2}(r) \leq(m+2) T(r, f)+O\left(r^{q-1}\right)+O(\log r)+S_{2}(r),
$$

as $r \notin E_{1} \cup E$ and $r \rightarrow+\infty$.

Since $f$ is a transcendental entire function, we deduce by (3.48) that:

$$
S_{2}(r)=o(T(r, f))+O\left(r^{q-1}\right),
$$

as $r \notin E_{1} \cup E$ and $r \rightarrow+\infty$.

On the other hand, by rewriting (3.35), we have:

$$
\frac{f^{n}(z)}{\gamma_{2}(z)}+\frac{p(z) f(z+\eta)}{\gamma_{2}(z)}=\sum_{j=1}^{m} H_{2, j}(z) e^{\omega_{j} z^{q}}
$$

By (3.33), (3.34), (3.43) and Definition 1.1, we deduce:

$$
\begin{aligned}
N\left(r, \frac{1}{\frac{f^{n}(z)}{\gamma_{2}(z)}+\frac{p(z) f(z+\eta)}{\gamma_{2}(z)}}\right) & \leq T\left(r, \frac{f^{n}(z)}{\gamma_{2}(z)}+\frac{p(z) f(z+\eta)}{\gamma_{2}(z)}\right)+O(1) \\
& \leq T\left(r, f^{n}(z)\right)+T(r, f(z+\eta))+O\left(r^{q-1}\right)+O(1) \\
& \leq T\left(r, f^{n}(z)\right)+T(r, f(z+\eta))+O\left(r^{q-1}\right)+O(1) \\
& \leq(n+1) T(r, f(z))+O\left(r^{q-1}\right)+o\left(\frac{T(r, f(z))}{r^{\delta}}\right),
\end{aligned}
$$

as $r \notin E_{1}$ and $r \rightarrow \infty$. Here, $\delta$ is the fixed positive number defined as in (3.43).

On the other hand, by (3.33), (3.34), (3.36), (3.50), Lemma 2.4, and the assumption $(\mathrm{H})$, we deduce:

$$
\begin{aligned}
N\left(r, \frac{1}{\frac{f^{n}(z)}{\gamma_{2}(z)}+\frac{p(z) f(z+\eta)}{\gamma_{2}(z)}}\right) & =N\left(r, \frac{1}{\sum_{j=1}^{m} H_{2, j}(z) e^{\omega_{j} z^{q}}}\right) \\
& =N\left(r, \frac{1}{H_{2,1}(z)+\sum_{j=2}^{m} H_{2, j}(z) e^{\left(\omega_{j}-\omega_{1}\right) z^{q}}}\right) \\
& =C\left(\operatorname{co}\left(\hat{W}_{0}\right)\right) \frac{r^{q}}{2 \pi}+o\left(r^{q}\right),
\end{aligned}
$$

where $\hat{W}_{0}$ is defined as in (3.29).

By (3.51) and (3.52), we deduce:

$$
C\left(\operatorname{co}\left(\hat{W}_{0}\right)\right) \frac{r^{q}}{2 \pi} \leq(n+1) T(r, f(z))+o\left(\frac{T(r, f(z))}{r^{\delta}}\right)+o\left(r^{q}\right),
$$


as $r \notin E_{1}$ and $r \rightarrow \infty$. Here, $\delta$ is the fixed positive number defined as in (3.43).

By dividing $f(z+\eta)$ on two sides of (3.35), we have:

$$
\frac{f^{n}(z)}{\gamma_{2}(z) f(z+\eta)}=\sum_{j=1}^{m} \frac{H_{2, j}(z) e^{\omega_{j} z^{q}}}{f(z+\eta)}-\frac{p(z)}{\gamma_{2}(z)} .
$$

By (3.33), (3.34), (3.36), (3.43), (3.53), (3.54), Lemma 2.4, and the assumption $n \geq m+2$, we deduce:

$$
\begin{aligned}
T\left(r, \frac{f^{n}(z)}{\gamma_{2}(z) f(z+\eta)}\right) \geq & T\left(r, f^{n}(z)\right)-T\left(r, \gamma_{2}(z) f(z+\eta)\right) \\
\geq & T\left(r, f^{n}(z)\right)-T(r, f(z+\eta))-T\left(r, \gamma_{2}(z)\right) \\
\geq & T\left(r, f^{n}(z)\right)-T(r, f(z))-T\left(r, \gamma_{2}(z)\right) \\
& +o\left(\frac{T(r, f(z))}{r^{\delta}}\right) \\
\geq & (n-1) T(r, f(z))+O\left(r^{q-1}\right)+o\left(\frac{T(r, f(z))}{r^{\delta}}\right) \\
\geq & \frac{n-1}{n+1} \cdot \frac{C\left(c o\left(\hat{W}_{0}\right)\right)}{2 \pi} r^{q}+o\left(r^{q}\right)
\end{aligned}
$$

and

$$
\begin{aligned}
& T\left(r, \frac{f^{n}(z)}{\gamma_{2}(z) f(z+\eta)}\right) \\
& \quad \leq T\left(r, \sum_{j=1}^{m} H_{2, j}(z) e^{\omega_{j} z^{q}}\right)+T\left(r, \frac{1}{f(z+\eta)}\right)+T\left(r,-\frac{p(z)}{\gamma_{2}(z)}\right) \\
& \quad \leq C\left(\operatorname{co}\left(\hat{W}_{0}\right)\right) \frac{r^{q}}{2 \pi}+T(r, f(z))+o\left(\frac{T(r, f(z))}{r^{\delta}}\right)+O\left(r^{q-1}\right)+O(\log r) \\
& \quad \leq C\left(\operatorname{co}\left(\hat{W}_{0}\right)\right) \frac{r^{q}}{2 \pi}+\frac{1}{n-1} T\left(r, \frac{f^{n}(z)}{\gamma_{2}(z) f(z+\eta)}\right)+o\left(r^{q}\right)
\end{aligned}
$$

as $r \notin E_{1}$ and $r \rightarrow \infty$.

By (3.55) and (3.56), we deduce:

$\frac{n-1}{n+1} \frac{C\left(c o\left(\hat{W}_{0}\right)\right)}{2 \pi} r^{q}+o\left(r^{q}\right) \leq T\left(r, \frac{f^{n}(z)}{\gamma_{2}(z) f(z+\eta)}\right) \leq \frac{n-1}{n-2} \frac{C\left(c o\left(\hat{W}_{0}\right)\right)}{2 \pi} r^{q}+o\left(r^{q}\right)$

and

$$
\frac{1}{n+1} \cdot \frac{C\left(c o\left(\hat{W}_{0}\right)\right)}{2 \pi} r^{q}+o\left(r^{q}\right) \leq T(r, f) \leq \frac{1}{n-2} \cdot \frac{C\left(\operatorname{co}\left(\hat{W}_{0}\right)\right)}{2 \pi} r^{q}+o\left(r^{q}\right),
$$

as $r \rightarrow \infty$ and $r \notin E_{1}$.

By (3.54). we consider the following two subcases.

Subcase 2.2.1.2.1. Suppose that there exists some subset $I \subset(0,+\infty)$ of infinite logarithmic measure, i.e., $\int_{I \cap[1,+\infty)} \frac{\mathrm{d} t}{t}=+\infty$, and there exists one 
and only one of the $m$ distinct meromorphic functions $\frac{H_{2,1}(z) e^{\omega_{1} z^{q}}}{f(z+\eta)}, \frac{H_{2,2}(z) e^{\omega_{2} z^{q}}}{f(z+\eta)}$, $\ldots, \frac{H_{2, m}(z) e^{\omega_{m} z^{q}}}{f(z+\eta)}$ in $(3.54)$, say $\frac{H_{2,1}(z) e^{\omega_{1} z^{q}}}{f(z+\eta)}$, such that:

$$
T\left(r, \frac{H_{2,1}(z) e^{\omega_{1} z^{q}}}{f(z+\eta)}\right) \leq A_{1} r^{q-1+\varepsilon},
$$

as $r \in I$ and $r \rightarrow+\infty$, while:

$$
T\left(r, \frac{H_{2, j}(z) e^{\omega_{j} z^{q}}}{f(z+\eta)}\right) \geq A_{j} r^{q} \quad \text { for } \quad 2 \leq j \leq m
$$

as $r \in I$ and $r \rightarrow+\infty$. Here, $A_{j}>0$ is a constant for $1 \leq j \leq m$. This together with (3.43), the assumption $(\mathrm{H})$, and Hayman [10, p.7], gives:

$$
A_{j} r^{q} \leq T\left(r, \frac{H_{2, j}(z) e^{\omega_{j} z^{q}}}{f(z+\eta)}\right) \leq \tilde{A}_{j} r^{q} \quad \text { for } \quad 2 \leq j \leq m
$$

as $r \in I$ and $r \rightarrow+\infty$. Here, $\tilde{A}_{j}$ is a positive number ,such that $A_{j}<\tilde{A}_{j}$ for $2 \leq j \leq m$.

By (3.33), (3.34), (3.42), (3.46)-(3.49), and (3.58), we deduce:

$$
T\left(r,-\frac{p(z)}{\gamma_{2}(z)}\right)+N\left(r, \frac{1}{f(z+\eta)}\right)+N\left(r, \frac{1}{f(z)}\right)=o(T(r, f(z))),
$$

as $r \notin E_{1} \cup E$ and $r \rightarrow+\infty$.

By (3.54), (3.57), (3.59), (3.61), and Lemma 2.1, we deduce that the positive integer $m$ satisfies $m \geq 2$.

By (3.57), (3.59), (3.60), and the assumption $n \geq m+2$, we deduce:

$$
T\left(r, \frac{H_{2,1}(z) e^{\omega_{1} z^{q}}}{f(z+\eta)}\right)=o\left(T\left(r, \frac{f^{n}(z)}{\gamma_{2}(z) f(z+\eta)}\right)\right)
$$

and

$$
T\left(r, \frac{H_{2,1}(z) e^{\omega_{1} z^{q}}}{f(z+\eta)}\right)=o\left(T\left(r, \frac{H_{2, j}(z) e^{\omega_{j} z^{q}}}{f(z+\eta)}\right)\right) \quad \text { for } \quad 2 \leq j \leq m
$$

as $r \in I$ and $r \rightarrow+\infty$.

By (3.57), (3.60), (3.61), and the assumption $n \geq m+2$, we deduce:

$$
T\left(r,-\frac{p(z)}{\gamma_{2}(z)}\right)=o\left(T\left(r, \frac{f^{n}(z)}{\gamma_{2}(z) f(z+\eta)}\right)\right)
$$

and

$$
T\left(r,-\frac{p(z)}{\gamma_{2}(z)}\right)=o\left(T\left(r, \frac{H_{2, j}(z) e^{\omega_{j} z^{q}}}{f(z+\eta)}\right)\right) \quad \text { for } \quad 2 \leq j \leq m,
$$

as $r \in I$ and $r \rightarrow+\infty$. 
By (3.33), (3.42), (3.47), (3.57), (3.58), (3.60), the assumption (H), and Hayman [10, p.7], we deduce:

$$
\begin{aligned}
& N\left(r, \frac{f^{n}(z)}{\gamma_{2}(z) f(z+\eta)}\right)+N\left(r, \frac{\gamma_{2}(z) f(z+\eta)}{f^{n}(z)}\right)+\sum_{j=2}^{m} N\left(r, \frac{H_{2, j}(z) e^{\omega_{j} z^{q}}}{f(z+\eta)}\right) \\
& \quad+\sum_{j=2}^{m} N\left(r, \frac{f(z+\eta)}{H_{2, j}(z) e^{\omega_{j} z^{q}}}\right)=o\left(T\left(r, \frac{f^{n}(z)}{\gamma_{2}(z) f(z+\eta)}\right)\right)
\end{aligned}
$$

and

$$
\begin{aligned}
& N\left(r, \frac{f^{n}(z)}{\gamma_{2}(z) f(z+\eta)}\right)+N\left(r, \frac{\gamma_{2}(z) f(z+\eta)}{f^{n}(z)}\right)+\sum_{j=2}^{m} N\left(r, \frac{H_{2, j}(z) e^{\omega_{j} z^{q}}}{f(z+\eta)}\right) \\
& \quad+\sum_{j=2}^{m} N\left(r, \frac{f(z+\eta)}{H_{2, j}(z) e^{\omega_{j} z^{q}}}\right)=o\left(T\left(r, \frac{H_{2, j}(z) e^{\omega_{j} z^{q}}}{f(z+\eta)}\right)\right),
\end{aligned}
$$

as $r \in I$ and $r \rightarrow+\infty$.

By (3.54), (3.62)-(3.67), and Lemma 2.1, we deduce:

$$
\frac{H_{2,1}(z) e^{\omega_{j} z^{q}}}{f(z+\eta)}-\frac{p(z)}{\gamma_{2}(z)} \equiv 0
$$

and

$$
\frac{f^{n}(z)}{\gamma_{2}(z) f(z+\eta)}=\sum_{j=2}^{m} \frac{H_{2, j}(z) e^{\omega_{j} z^{q}}}{f(z+\eta)}
$$

and so, it follows by (3.36) that:

$$
f(z+\eta)=\frac{\gamma_{2}(z) H_{2,1}(z) e^{\omega_{1} z^{q}}}{p(z)}=\frac{H_{1}(z) e^{\omega_{1} z^{q}}}{p(z)}
$$

and

$$
f^{n}(z+\eta)=\gamma_{2}(z+\eta) \sum_{j=2}^{m} H_{2, j}(z+\eta) e^{\omega_{j}(z+\eta)^{q}}=\sum_{j=2}^{m} H_{j}(z+\eta) e^{\omega_{j}(z+\eta)^{q}}
$$

By substituting (3.68) into (3.69), we deduce:

$$
H_{1}^{n}(z) e^{n \omega_{1} z^{q}}=p^{n}(z) \sum_{j=2}^{m} L_{j}(z) e^{\omega_{j} z^{q}}
$$

where

$$
L_{j}(z)=H_{j}(z+\eta) e^{\omega_{j} P_{q-1}(z)} \quad \text { for } \quad 2 \leq j \leq m
$$

Here:

$$
P_{q-1}(z)=\sum_{k=1}^{q}\left(\begin{array}{l}
q \\
k
\end{array}\right) \eta^{k} z^{q-k}
$$


By (3.71), (3.72), Lemma 2.6, and Hayman [10, p.7], we deduce:

$$
\begin{aligned}
T\left(r, L_{j}(z)\right) & \leq T\left(r, H_{j}(z+\eta)\right)+T\left(r, e^{\omega_{j} P_{q-1}(z)}\right) \\
& =T\left(r, H_{j}(z)\right)+T\left(r, e^{\omega_{j} P_{q-1}(z)}\right)+O\left(r^{q-2+\varepsilon}\right)+O(\log r) \\
& \leq T\left(r, e^{\omega_{j} P_{q-1}(z)}\right)+O\left(r^{q-2+\varepsilon}\right)+O\left(r^{q-1}\right)+O(\log r) \\
& =\frac{q|\eta| r^{q-1}}{\pi}(1+o(1))+O\left(r^{q-2+\varepsilon}\right)+O\left(r^{q-1}\right)+O(\log r)=o\left(r^{q}\right)
\end{aligned}
$$

for $2 \leq j \leq m$, as $r \rightarrow+\infty$.

We consider the following two subcases.

Subcase 2.2.1.2.1.1. Suppose that $m=2$. Then, (3.70) can be rewritten as:

$$
e^{\left(n \omega_{1}-\omega_{2}\right) z^{q}}=\frac{p^{n}(z) L_{2}(z)}{H_{1}^{n}(z)} .
$$

By (3.71), (3.73), (3.74), the assumption $(\mathrm{H})$, and Hayman [10, p.7], we deduce:

$$
T\left(r, \frac{p^{n} L_{2}}{H_{1}^{n}}\right)=O\left(r^{q-1}\right)+O(\log r)
$$

and

$$
T\left(r, H_{1}^{n}\right)+T\left(r, p^{n} L_{j}\right)=O\left(r^{q-1}\right)+O(\log r) \quad \text { for } \quad 2 \leq j \leq m .
$$

By (3.74), (3.75) and Hayman [10, p.7], we deduce:

$$
\begin{aligned}
\frac{\left|n \omega_{1}-\omega_{2}\right|}{\pi} r^{q}(1+o(1)) & =T\left(r, e^{\left(n \omega_{1}-\omega_{2}\right) z^{q}}\right)=T\left(r, \frac{p^{n}(z) L_{2}(z)}{H_{1}^{n}(z)}\right) \\
& =O\left(r^{q-1}\right)+O(\log r),
\end{aligned}
$$

which implies that $\omega_{2}=n \omega_{1}$. This together with (3.70)-(3.72) implies that:

$$
H_{1}^{n}(z)=p^{n}(z) H_{2}(z+\eta) e^{\omega_{2} P_{q-1}(z)},
$$

where $P_{q-1}(z)$ is defined as in (3.72).

By (3.68), (3.72), (3.77), and $\omega_{2}=n \omega_{1}$, we get the conclusion (i) of Theorem 1.8. 
Subcase 2.2.1.2.1.2. Suppose that $m \geq 3$. Then, by (3.70), (3.76), Lemma 2.4 and the fact $p^{n} L_{j} \not \equiv 0$ for $2 \leq j \leq m$, we deduce:

$$
\begin{aligned}
C\left(\operatorname{co}\left(\tilde{W}_{0}\right)\right) \frac{r^{q}}{2 \pi}+o\left(r^{q}\right) & =N\left(r, \frac{1}{p^{n}(z) L_{2}(z)+p^{n}(z) \sum_{j=3}^{m} L_{j}(z) e^{\left(\omega_{j}-\omega_{2}\right) z^{q}}}\right) \\
& =N\left(r, \frac{1}{p^{n}(z) \sum_{j=2}^{m} L_{j}(z) e^{\omega_{j} z^{q}}}\right)=N\left(r, \frac{1}{H_{1}^{n}(z) e^{n \omega_{1} z^{q}}}\right) \\
& =N\left(r, \frac{1}{H_{1}^{n}(z)}\right) \\
& \leq T\left(r, H_{1}^{n}(z)\right)+O(1)=O\left(r^{q-1}\right)+O(\log r)+O(1) \\
& =o\left(r^{q}\right),
\end{aligned}
$$

which is impossible. Here, $\tilde{W}_{0}=\left\{0, \overline{w_{3}-w_{2}}, \overline{w_{4}-w_{2}}, \ldots, \overline{w_{m}-w_{2}}\right\}$.

Subcase 2.2.1.2.2. Suppose that there exists some subset $I \subset(0,+\infty)$ with infinite logarithmic measure, i.e., $\int_{I \cap[1,+\infty)} \frac{\mathrm{d} t}{t}=+\infty$, and there exist at least two of the $m$ distinct meromorphic functions $\frac{H_{2,1}(z) e^{\omega_{1} z^{q}}}{f(z+\eta)}, \frac{H_{2,2}(z) e^{\omega_{2} z^{q}}}{f(z+\eta)}$, $\ldots, \frac{H_{2, m}(z) e^{\omega_{m} z^{q}}}{f(z+\eta)}$ in (3.54), say $\frac{H_{2,1}(z) e^{\omega_{1} z^{q}}}{f(z+\eta)}$ and $\frac{H_{2,2}(z) e^{\omega_{2} z^{q}}}{f(z+\eta)}$, such that (3.59) holds, such that (3.60) holds for $3 \leq j \leq m$, and such that:

$$
T\left(r, \frac{H_{2,2}(z) e^{\omega_{2} z^{q}}}{f(z+\eta)}\right) \leq B_{1} r^{q-1+\varepsilon},
$$

as $r \in I$ and $r \rightarrow+\infty$. Here, $B_{1}>0$ is a constant.

By (3.36), (3.59), (3.78), the assumption (H), and Hayman [10, p.7], we deduce:

$$
\begin{aligned}
\frac{\left.\mid \omega_{1}-\omega_{2}\right) \mid}{\pi} r^{q}(1+o(1))= & T\left(r, \frac{H_{1}(z)}{H_{2}(z)} e^{\left(\omega_{1}-\omega_{2}\right) z^{q}}\right)+O\left(r^{q-1}\right)+O(\log r) \\
= & T\left(r, \frac{\frac{H_{2,1}(z) e^{\omega_{1} z^{q}}}{f(z+\eta)}}{\frac{H_{2,2}(z) e^{\omega_{2} z^{q}}}{f(z+\eta)}}\right)+O\left(r^{q-1}\right)+O(\log r) \\
\leq & T\left(r, \frac{H_{2,1}(z) e^{\omega_{1} z^{q}}}{f(z+\eta)}\right)+T\left(r, \frac{H_{2,2}(z) e^{\omega_{2} z^{q}}}{f(z+\eta)}\right) \\
& +O\left(r^{q-1}\right)+O(\log r)+O(1) \\
\leq & A_{1} r^{q-1+\varepsilon}+B_{1} r^{q-1+\varepsilon}+O\left(r^{q-1}\right)+O(\log r)+O(1) \\
& =o\left(r^{q}\right),
\end{aligned}
$$

as $r \in I$ and $r \rightarrow+\infty$. This is impossible. 
Subcase 2.2.1.2.3. Suppose that the $m$ distinct meromorphic functions $\frac{H_{2,1}(z) e^{\omega_{1} z^{q}}}{f(z+\eta)}, \frac{H_{2,2}(z) e^{\omega_{2} z^{q}}}{f(z+\eta)}, \ldots, \frac{H_{2, m}(z) e^{\omega_{m} z^{q}}}{f(z+\eta)}$ in (3.54) are such that:

$$
T\left(r, \frac{H_{2, j}(z) e^{\omega_{j} z^{q}}}{f(z+\eta)}\right) \geq C_{j} r^{q} \quad \text { for } \quad 1 \leq j \leq m
$$

as $r \notin E_{1}$ and $r \rightarrow+\infty$. Here, $C_{j}>0$ is a constant for $1 \leq j \leq m$.

On the other hand, by (3.34), (3.36), (3.43), (3.58), the assumption (H), and Hayman [10, p.7], we deduce:

$$
\begin{aligned}
T\left(r, \frac{H_{2, j}(z) e^{\omega_{j} z^{q}}}{f(z+\eta)}\right) \leq & T\left(r, H_{2, j}(z) e^{\omega_{j} z^{q}}\right)+T\left(r, \frac{1}{f(z+\eta)}\right) \\
\leq & \frac{\left|\omega_{j}\right|}{\pi} r^{q}(1+o(1))+T(r, f(z+\eta))+O\left(r^{q-1}\right)+O(\log r) \\
\leq & \frac{\left|\omega_{j}\right|}{\pi} r^{q}(1+o(1))+T(r, f(z))+o\left(\frac{T(r, f(z))}{r^{\delta}}\right) \\
& +O\left(r^{q-1}\right)+O(\log r) \\
\leq & \left(\frac{\left|\omega_{j}\right|}{\pi}+\frac{1}{n-2} \cdot \frac{C\left(c o\left(\hat{W}_{0}\right)\right)}{2 \pi}\right) r^{q}(1+o(1))+o\left(r^{q}\right)
\end{aligned}
$$

for $1 \leq j \leq m$, as $r \notin E_{1}$ and $r \rightarrow+\infty$.

By (3.79) and (3.80), we deduce:

$C_{j} r^{q} \leq T\left(r, \frac{H_{2, j}(z) e^{\omega_{j} z^{q}}}{f(z+\eta)}\right) \leq\left(\frac{\left|\omega_{j}\right|}{\pi}+\frac{1}{n-2} \cdot \frac{C\left(c o\left(\hat{W}_{0}\right)\right)}{2 \pi}\right) r^{q}(1+o(1))+o\left(r^{q}\right)$

for $1 \leq j \leq m$, as $r \notin E_{1}$ and $r \rightarrow+\infty$.

Next, in the same manner as in Subcase 2.2.1.2.1, we can deduce by (3.57) and (3.81) that:

$$
\begin{aligned}
& T\left(r,-\frac{p(z)}{\gamma_{2}(z)}\right)=o\left(T\left(r, \frac{f^{n}(z)}{\gamma_{2}(z) f(z+\eta)}\right)\right) \\
& T\left(r,-\frac{p(z)}{\gamma_{2}(z)}\right)=o\left(T\left(r, \frac{H_{2, j}(z) e^{\omega_{j} z^{q}}}{f(z+\eta)}\right)\right) \text { for } \quad 1 \leq j \leq m, \\
& N\left(r, \frac{f^{n}(z)}{\gamma_{2}(z) f(z+\eta)}\right)+N\left(r, \frac{\gamma_{2}(z) f(z+\eta)}{f^{n}(z)}\right)+\sum_{k=1}^{m} N\left(r, \frac{H_{2, k}(z) e^{\omega_{k} z^{q}}}{f(z+\eta)}\right) \\
& \quad+\sum_{k=1}^{m} N\left(r, \frac{f(z+\eta)}{H_{2, k}(z) e^{\omega_{k} z^{q}}}\right)=o\left(T\left(r, \frac{f^{n}(z)}{\gamma_{2}(z) f(z+\eta)}\right)\right)
\end{aligned}
$$

and 


$$
\begin{aligned}
& N\left(r, \frac{f^{n}(z)}{\gamma_{2}(z) f(z+\eta)}\right)+N\left(r, \frac{\gamma_{2}(z) f(z+\eta)}{f^{n}(z)}\right)+\sum_{k=1}^{m} N\left(r, \frac{H_{2, k}(z) e^{\omega_{k} z^{q}}}{f(z+\eta)}\right) \\
& \quad+\sum_{k=1}^{m} N\left(r, \frac{f(z+\eta)}{H_{2, k}(z) e^{\omega_{k} z^{q}}}\right)=o\left(T\left(r, \frac{H_{2, j}(z) e^{\omega_{j} z^{q}}}{f(z+\eta)}\right)\right) \quad \text { for } \quad 1 \leq j \leq m,
\end{aligned}
$$

as $r \notin E_{1}$ and $r \rightarrow+\infty$.

By (3.54), (3.82)-(3.85), Lemma 2.1, and the supposition that the $m+1$ entire functions in (3.31) are linearly independent in the complex plane, we deduce that $-\frac{p}{\gamma_{2}}=0$, which is impossible.

Suppose that $f^{n}(z)$ and the $m+1$ distinct entire functions in (3.31) have at most finitely many distinct nonzero common zeros in the complex plane, say $a_{2,1}, a_{2,2}, \ldots, a_{2, l_{2}}$ are all the distinct nonzero common zeros of $f^{n}(z)$ and the $m+1$ distinct entire functions of (3.31) in the complex plane, where $l_{2}$ is a positive integer, and each such common zero $a_{2, j} \in\left\{a_{2, j}\right\}_{j=1}^{l_{2}}$ is counted according to the minimum positive integer $\tilde{m}_{2, j}$ of all the multiplicities of $a_{2, j}$ as the zero of $f^{n}(z)$ and the $m+1$ distinct entire functions of (3.31), respectively. Now, we let:

$$
P_{1,2}(z)=z^{m_{2,0}} \prod_{j=1}^{l_{2}}\left(z-a_{2, j}\right)^{\tilde{m}_{2, j}},
$$

where $m_{2,0}$ is the nonnegative integer that is defined as in (3.32). Next, we replace the polynomial $P_{1,2}$ defined as in (3.86) with the entire function $\gamma_{2}$ defined as in (3.32), and then use the lines as the above, we can get a contradiction.

Subcase 2.2.2. Suppose that $H_{0} \not \equiv 0$. Then, (1.1) can be rewritten as:

$$
f^{n}(z)=H_{0}(z)+H_{1}(z) e^{\omega_{1} z^{q}}+H_{2}(z) e^{\omega_{2} z^{q}}+\cdots+H_{m}(z) e^{\omega_{m} z^{q}}-p(z) f(z+\eta) .
$$

By (3.87), we deduce that either that the $m+2$ entire functions:

$$
\begin{aligned}
& H_{0}(z), H_{1}(z) e^{\omega_{1} z^{q}}, H_{2}(z) e^{\omega_{2} z^{q}}, \ldots, H_{m-1}(z) e^{\omega_{(m-1)} z^{q}}, H_{m}(z) e^{\omega_{(m)} z^{q}}, \\
& \quad-p(z) f(z+\eta)
\end{aligned}
$$

are $m+2$ linearly dependent entire functions in the complex plane, or that the $m+2$ entire functions in (3.88) are linearly independent entire functions in the complex plane.

Next, in the same manner as in the proof of Subcase 2.2.1.1 and Subcase 2.2.1.2, by Lemmas 2.5 and 2.6, we deduce (3.42) and (3.43). By (3.42), (3.43), (3.87), Lemma 2.4, and the supposition $H_{0} \not \equiv 0$, we deduce $n=m+2$,

$$
\frac{C\left(\operatorname{co}\left(W_{0}\right)\right)}{2(n+1) \pi} r^{q}+o\left(r^{q}\right) \leq T(r, f(z)) \leq \frac{C\left(\operatorname{co}\left(W_{0}\right)\right)}{2(n-1) \pi} r^{q}+o\left(r^{q}\right)
$$

and

$$
\begin{aligned}
& B_{2} T(r, f(z)) \leq N\left(r, \frac{1}{f(z)}\right) \leq T(r, f)+O(1), \quad \text { as } \quad r \notin E_{1} \cup E \quad \text { and } \\
& \quad r \rightarrow+\infty
\end{aligned}
$$


Here $B_{2}$ is some positive constant.

By Definition 1.1 and the standard reasoning of removing an exceptional set (cf. [12, Lemma 1.1.2]). we deduce by (3.89) and (3.90) that:

$$
\lambda(f)=\rho(f)=q .
$$

By (3.91) and the obtained result $n=m+2$, we get the conclusion (ii) of Theorem 1.8. This completes the proof of Theorem 1.8.

\section{Acknowledgements}

The authors wish to express their thanks to the referee for his/her valuable suggestions and comments. The first author of this paper also wants to express his sincere thanks to Professor R. Korhonen, Professor J. Heittokangas, and Professor I. Laine for their help and guidance during his visit at the Department of Physics and Mathematics, University of Eastern Finland from June 10, 2019 to August 20, 2019. This sped up the completion of this article.

Open Access. This article is licensed under a Creative Commons Attribution 4.0 International License, which permits use, sharing, adaptation, distribution and reproduction in any medium or format, as long as you give appropriate credit to the original author(s) and the source, provide a link to the Creative Commons licence, and indicate if changes were made. The images or other third party material in this article are included in the article's Creative Commons licence, unless indicated otherwise in a credit line to the material. If material is not included in the article's Creative Commons licence and your intended use is not permitted by statutory regulation or exceeds the permitted use, you will need to obtain permission directly from the copyright holder. To view a copy of this licence, visit http:// creativecommons.org/licenses/by/4.0/.

Publisher's Note Springer Nature remains neutral with regard to jurisdictional claims in published maps and institutional affiliations.

\section{References}

[1] Ash, R.B.: Complex variables. Academic Press, New York (1971)

[2] Ahlfors, L.: Complex Analysis. McGraw-Hill, New York (1979)

[3] Cartan, H.: Sur les zéros des combinaisons linéaires de $p$ fonctions holomorphes données. Mathematica Cluj 7, 5-31 (1933)

[4] Chen, Z.X.: Complex Differences and Difference Equations. Science Press, Beijing (2014)

[5] Chiang, Y.M., Feng, S.J.: On the Nevanlinna characteristic of $f(z+\eta)$ and difference equations in the complex plane. Ramanujan J. 16(1), 105-129 (2008)

[6] Gundersen, G.G., Hayman, W.K.: The strength of Cartan's version of Nevanlinna theory. Bull. Lond. Math. Soc. 36(4), 433-454 (2004)

[7] Halburd, R.G., Korhonen, R.J.: Nevanlinna theory for the difference operator. Ann. Acad. Sci. Fenn. Math. 31(2), 463-478 (2006)

[8] Halburd, R.G., Korhonen, R.J.: Difference analogue of the lemma on the logarithmic derivative with applications to difference equations. J. Math. Anal. Appl. 314(2), 477-487 (2006) 
[9] Halburd, R.G., Korhonen, R.J., Tohge, K.: Holomorphic curves with shiftinvariant hyperplane preimages. Trans. Am. Math. Soc. 366(8), 4267-4298 (2014)

[10] Hayman, W.K.: Meromorphic Functions. Clarendon Press, Oxford (1964)

[11] Heittokangas, J., Ishizaki, K., Tohge, K., Wen, Z.T.: Zero distribution and division results for exponential polynomials. Isr. J. Math. 227(1), 397-421 (2018)

[12] Laine, I.: Nevanlinna Theory and Complex Differential Equations. Walter de Gruyter, Berlin (1993)

[13] Latreuch, Z.: On the existence of entire solutions of certain class of nonlinear difference equations. Mediterr. J. Math. 14(3), 115 (2017)

[14] Steinmetz, N.: Zur Wertverteilung von Exponentialpolynomen. Manuscr. Math. 26(1-2), 155-167 (1978/79)

[15] Yang, C.C., Yi, H.X.: Uniqueness Theory of Meromorphic Functions. Kluwer Academic Publishers, Dordrecht (2003)

[16] Yang, C.C., Laine, I.: On analogies between nonlinear difference and differential equations. Proc. Jpn. Acad. Ser. A 86(1), 10-14 (2010)

[17] Yang, L.: Value Distribution Theory. Springer, Berlin (1993)

[18] Zhang, R.R., Chen, Z.X.: Fixed points of meromorphic functions and of their differences, divided differences and shifts. Acta. Math. Sin. (Engl. Ser.) 32(10), 1189-1202 (2016)

[19] Zhang, R.R., Huang, Z.B.: On meromorphic solutions of non-linear difference equations. Comput. Methods Funct. Theory 18(3), 389-408 (2018)

Xiao-Min Li and Chen-Shuang Hao

Department of Mathematics

Ocean University of China

Qingdao 266100

Shandong

People's Republic of China

e-mail: lixiaomin@ouc.edu.cn

Chen-Shuang Hao

e-mail: 563825943@qq.com

Hong-Xun Yi

Department of Mathematics

Shandong University

Jinan 250199

Shandong

People's Republic of China

e-mail: hxyi@sdu.edu.cn

Received: October 7, 2019.

Revised: July 21, 2020.

Accepted: December 23, 2020. 